\title{
Primary central nervous system vasculitis: a review of this challenging diagnosis
}

\author{
Oliveira FTM* and Pessoa LFO \\ Department of Neurology, Santa Casa at São Paulo-SP, Ambulatory of neurological manifestations of systemic diseases, Brazil
}

\begin{abstract}
The primary vasculitis of the central nervous system is still a controversial subject and in constant study for a better understanding of all its aspects. It is a pathophysiology disease that is not yet fully understood, and with probable immunological and biological mechanisms of very high complexity, which go beyond the current technical and scientific limits. Its clinical manifestations are challenging even for the most experienced neurologists, which requires, in addition to academic knowledge, a unique ability to interpret clinically and to weigh the results presented in complementary tests. This is because we do not have any non-invasive diagnostic tool that confirms, or discards, with full security, the possibility of VPSNC. In practice, the confirmatory examination is considered the gold standard and is still the brain biopsy, which is usually performed only in exceptional cases.
\end{abstract}

\section{Introduction}

Primary Central Nervous System Vasculitis (VPSNC) is a rare and poorly defined clinical condition, which began to be better understood in the late 1950's, when pathological studies performed by Cravioto and Feigin [1] described some associated neurological events to an inflammatory process restricted to the brain and spinal cord of granulomatous histological characteristics. It was initially referred to as idiopathic or non-inflammatory granulomatous arteritis of the CNS, or giant cell arteritis of the CNS. At that time, such condition was related to usually unfavorable developments, usually with outcomes with moderate to severe disability or death, despite the available treatments, which due to the lack of studies based on clinical trials, used to be nonstandardized and had no scientific basis to support the rationalization of its uses.

Over time, the understanding of VPSNC-related conditions has expanded significantly, as well as the clarification of other conditions with clinical presentation and similar pathophysiological features, but which can now be understood as distinct diseases, such as, for example, reversible cerebral vasoconstriction syndrome and reversible posterior encephalopathy syndrome. In addition, the development of diagnostic techniques, both for laboratory analysis and increasingly accurate and accurate imaging methods, have helped to build the pathophysiological details of the NSDS.

The treatment protocols for this condition are not yet standardized and are generally elaborated based on diseases of similar pathophysiological understanding, such as systemic vasculitis and immune-mediated diseases. However, we already have well-developed cohort studies, designed by specialized centers, with an emphasis on diagnosis and treatment, which have been modifying the evolutionary course of the disease, transforming the vital and functional prognosis of the patients affected by this disease.

This work aims to carry out a thematic review of the current literature, based on recent work and carried out by specialized study centers.

\section{Method}

The research conducted in this study was carried out essentially with the help of the PubMed portal (https://www.ncbi.nlm.nih.gov/ pubmed/), managed by the US National Institute of Health (NIH).

The terms "Primary angiitis of Central Nervous System", "Vasculitis in Central Nervous System", were inserted for research. Articles with a publication dating from 2005 to 2017, including also articles of historical relevance, and published in indexed journals and considered as publications of high academic impact, were selected for consultation. It was decided to give greater emphasis to articles related to primary vasculitis, although discussions related to secondary vasculitis cannot be completely ignored. The study of the involvement in adults was prioritized, since the NSVC has particular characteristics in the pediatric population, besides being usually excluded from the sampling of the largest and most important studies.

\section{Primary vasculitis of the central nervous system \\ Definition}

Primary Vasculitis of the Central Nervous System is a rare condition, with an estimated annual incidence of 2.4 per 1,000,000 ha / year $(95 \%$ CI: 0.7-4.1). There are two main cohorts performed in adults, one in North America with 163 patients [2] and the other in France, which included 102 patients [3]. In both, the distribution is similar between men and women, therefore, there is no gender preference. The disease may present in any age group, and the involvement in children seems to have different physiopathology from that described in adults, with evidence of different lymphocytic lines mediating the inflammatory

Correspondence to: Oliveira, FTM. Department of Neurology, Santa Casa at São Paulo- SP, Av. Dr Cesario Mota Jr, 112 - V. Buarque. São Paulo-SP, Brazil, CEP 01221-010. E-mail: towmaz@gmail.com

Received: September 27, 2017; Accepted: October 23, 2017; Published: October 24,2017 
process, and generating particularities in the classification and evolution of the disease in this population [4]. In studies performed in adults the mean age of presentation was between 46 and 48 years, and patients aged 17 to 85 years were included. No related study in these studies referred to ethnic predominance in the CNPSV.

\section{Pathophysiology}

The pathophysiology of the disease is still not well understood. There is evidence that it is a parainfectious phenomenon, triggered by microorganisms through molecular mimicry, generating an antigenspecific immune response, with an inflammatory process in the walls of cerebral vessels by infiltration of CD4 + T lymphocytes, memory cells of the cellular immune system [5]. In children with CNPSV, evidence in immunohistochemical biopsy studies shows an inflammatory infiltrate with a predominance of cytotoxic CD8 + T lymphocytes [6]. Several microorganisms have already been implicated in the immunogenic trigger mechanism of NPVSV. In experimental studies, the VaricellaZoster virus, and subspecies of Mycoplasma sp. were the most related [7,8].

Another pathophysiological evidence is the frequent correlation of NSVCV with the vasculitic form of Cerebral Amyloid Angiopathy. It is believed that erroneous cleavage of the amyloid precursor protein generates oligomeric structures of low solubility. These structures are deposited in vascular tissues, and can trigger inflammatory cascades with release of kinins, complement activation, oxidative stress and changes in the blood-brain barrier, which are histopathologically translated with granulomatous transmural angiitis pattern [9].

\section{Histopathology}

Histopathologically there are 3 well-characterized patterns in NPVN: granulomatous, necrotizing and lymphocytic. The granulomatous pattern shows a vasculocentric mononuclear infiltrate surrounded by multinucleated cells. It is related in half the cases to the vasculitic form of amyloid angiopathy, from which the $\beta$-amyloid deposit can be evidenced. The pattern of necrotizing vasculitis is characterized by fibrinoid transmural necrosis, similar to that found in polyarteritis nodosa, and is the least common of the three patterns, and may be encountered rarely concomitantly with the granulomatous pattern. Finally, the lymphocytic pattern is characterized by lymphocytic infiltrate, with possible presence of plasma cells and vascular destruction [10]. There was divergence regarding the prevalence of histopathological patterns when compared to the two main cohorts performed until then $[2,3]$. The US study was categorical in stating that the granulomatous pattern was the most prevalent and was found in about $59 \%$ of its sample of 81 biopsies. The French study presented the lymphocytic pattern as the most prevalent, found in $84 \%$ of its sample of 49 biopsies. In both the necrotizing pattern was the least prevalent, accounting for $14 \%$ and $38 \%$, respectively.

In recent years, there has been a tendency to subdivide patients into two large groups, according to the size of the vessels affected by the disease. The VPSNC of medium and large vessels, characterized by imaging with characteristic lesions in intracranial, vertebral, basilar and proximal branches of the anterior, middle and posterior cerebral arteries; and VPSNC isolated from small vessels, with evidence of lesions only in second order divisions in front of the main intracranial arteries, or patients with normal imaging tests, but with diagnostic confirmation by biopsy. The importance of this subclassification lies in the fact that the evidence shows that the subtypes represent distinct patterns, both in relation to clinical and laboratory presentation, as to the evolutionary aspect, therapeutic response and vital and functional prognosis $[2,11]$.
In the cohorts performed, the predominance in the prevalence of the medium and large vessels pattern was well characterized, accounting for about 60 to $70 \%$ of the cases of NPSVD [2,3]. A subgroup of fast and progressive focal deficits is still considered within this group, being considered the subgroup of worse vital and functional prognosis, also correlating with granulomatous and / or necrotizing histopathological patterns [12].

\section{Clinical manifestations}

Clinical manifestations are extremely diverse, both in relation to the mode and time of presentation, and efforts have been made in recent years to relate characteristic syndromic conditions with pathophysiological subtypes of the disease. The presentation is usually insidious, and diagnostic time was realized in the first 6 months of symptoms in $75 \%$ of cases [10], but it may present acutely, including as an etiologic cause of an ischemic cerebrovascular event. The most prevalent symptom was headache, present in about $63 \%$ of cases, and the pattern may be variable, from localized headache, to a holocranial presentation, with a progressive tendency, but may also be remitting [10]. The American cohort reports that there was no record of presentation with sudden headache, or thunderclap pattern [2]. The information is relevant, since sudden headache is the usual presentation of reversible cerebral vasoconstriction syndrome, the main differential diagnosis of CNPSV. Cognitive decline was considered the second most common manifestation, being present in about half of the samples, but relating more closely to the NSVCV isolated from small vessels. Third, there are focal neurological deficits, more commonly with hemiparesis, but also with aphasia, ataxias, anopsias, diplopias and others $[4,13,14]$. Secondary ischemic events, usually abrupt, are present, and present mainly in the VPSNC of medium and large vessels (about $88 \%$ of these cases). Convulsive seizures are uncommon, present in less than $30 \%$ of the total cases, but when present, and in the presence of a suggestive primary vasculitis, it is very specific for the diagnosis of vasculitis isolated from small vessels present in $77 \%$ of these, and only in $21 \%$ of the vasculitis of medium and large vessels of CNS [11]. Other neurological manifestations are uncommon and nonspecific.

\section{Diagnosis}

The diagnostic criteria were proposed by Calabrese and Mallek in 1988 [2], based on the presence of 3 mandatory criteria:

1) New focal or diffuse neurological deficit and / or psychiatric symptoms, as compatible clinical picture;

2) Evidence of CNS vasculitis by angiography or biopsy, as a subsidiary examination;

3) Absence of evidence of systemic condition that may mimic or simulate CNS vasculitis, as a necessity to exclude differential diagnoses.

It is still considered today that the biopsy is the gold standard examination for the diagnosis, but it must be remembered that the vascular involvement has a segmental behavior, which makes its sensitivity limited. Biopsy studies guided by imaging studies show a significant gain in sensitivity (from 63 to $78 \%$ ) when biopsy is directed by such methods [15]. Preference should be given to biopsies in nondominant lobe, although the estimated risk of secondary sequelae is around $1 \%$ when performed by an experienced neurosurgeon [16].

The accuracy of digital angiography is indeterminate, since isolated small vessel disease may present with absolutely normal angiography. In addition, the characteristic changes of VPSNC are non-specific 
and may be similarly observed under different conditions, such as reversible cerebral vasoconstriction syndrome or even atherosclerosis. Nevertheless, it is considered the best imaging method and is necessary when brain angio-MRI or angio-CT is inconclusive if we are faced with a suggestive picture. The characteristic findings are the presence of segments with alternating stenosis and dilatations, determining bearding patterns, or delayed arterial filling and anastomotic channels [17], which may be unilateral or bilateral. Changes in MRI of the brain are very sensitive, but not very specific. Ischemic lesions are related to vasculitis of medium and large vessels, while the presence of leptomeningeal enhancement with gadolinium, or, more rarely, a tumefactive lesion, may be compatible with vasculitis isolated from small CNS vessels.

CSF changes are present in 80 to $90 \%$ of cases, but are also nonspecific, generally revealing a slight increase in leukocyte count and protein concentration [18]. The presence and intensity of CSF changes were also related to the vasculitis pattern isolated from small vessels in studies with the French cohort [11].

General laboratory tests do not usually aid in diagnosis. Interestingly, evidence of inflammatory activity, such as VHS and CRP, is normal in NPVC. This data is supported by the theory that the inflammatory process is absolutely restricted to the CNS and cannot extrapolate the limits of the blood-brain barrier, even if a significant barrier break is eventually evident. Similarly, constitutional symptoms of systemic vasculitis such as adynamia, fever, weight loss and others are usually absent in this clinical setting. Autoantibodies are also generally negative and do not relate to disease [18].

\section{Treatment}

There is no randomized clinical trial in the treatment of VPSNC. The strategies that are used are based on analogies made with the observed responses in systemic vasculitis, and in observational studies that have reported effectiveness in the treatment with glucocorticoid associated with cyclophosphamide [19].

Salvarani et al. (2015) suggest an induction regimen using Prednisone $1 \mathrm{mg} / \mathrm{kg} /$ day, in combination with cyclophosphamide, oral or daily, intravenously, in pulse therapy monthly for 3 to 6 months, followed by maintenance therapy with immunosuppressants, such as azathioprine, methotrexate or mycophenolate, for up to 18 months. For patients with acute presentation it is suggested induction in pulse therapy, with methylprednisolone $1 \mathrm{~g}$ / day for 3 to 5 days. About $27 \%$ of patients presented recurrence, and only $4 \%$ had 3 or more episodes of recurrence of symptoms.

Patients with medium and large vessel involvement were associated with a higher mortality risk and worse functional prognosis. Boysson et al. [11] reported conflicting data in their prospective cohort, emphasizing that they chose to use more aggressive treatments, with a preference for the combined use of prednisone with cyclophosphamide ( $80 \%$ vs. $44 \%$ in Salvarani et al, 2015), and induction and maintenance periods (mean of 24 months versus 9 months in Salvarani et al, [2]). There was no difference in mortality or functionality between the medium and large vessel groups when compared to the isolated group of small vessels, but this presented a higher recurrence of symptoms. It was also considered that the difference between the studies can be attributed to the predominance of the lymphocytic histopathological pattern in this study, since the best prognosis of this subgroup had been observed in Saveani et al (2007). [20]

\section{Case report}

A 29-year-old female patient started partial motor seizures with secondary generalization at 21 , which showed improvement after treatment with anticonvulsants. Associated with this, presented chronic holocranial headache, in tightening, with progressive worsening. After 5 years, he developed right hemiparesis and worsened motor coordination, associated with an increase in the frequency of seizures. Nuclear magnetic resonance imaging (MRI) revealed left brain hemiatrophy with multiple areas of contrast impregnation. Spectroscopy revealed minimal increase of choline peak and myoinositol with preservation of $\mathrm{N}$-acetylaspartate levels. During outpatient follow-up, at one year, he developed cognitive deterioration and worsened motor deficit. The hypothesis of primary vasculitis of the central nervous system was established and confirmed with left frontal lobe biopsy (perivascular lymphocytic infiltrate). Rheumatologic and infectious tests were negative. Treatment with corticosteroids and cyclophosphamide was initiated, and Rituximab was subsequently introduced, with significant improvement in symptoms and good control of epilepsy (Figure 1).

\section{Conclusion}

Because it is a rare clinical condition, it is a disease that is difficult to carry out a well-structured, multicenter scientific study where the individual contribution of each research center can contribute to the

Headache with characteristics of secondary involvement or alarm signals; rapidly progressive cognitive decline; focal neurological deficits; seizures

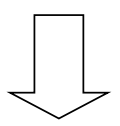

If there are systemic conditions that justify the clinical manifestation, proceed with specific treatment.

If there is no identifiable causal factor, continue imaging tests: Brain MRI and / or cerebral angiography (with or without brain biopsy)

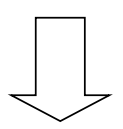

Diagnosis of Primary Vasculitis of the CNS (fulfills the criteria):

- Induction therapy: Methylprednisolone 1g EV 3-5 days +/Cyclophosphamide $0.75 \mathrm{~g} / \mathrm{m} 2$ per month / for 6 months

- Maintenance Therapy: Prednisone $1 \mathrm{mg} / \mathrm{kg} /$ day, initially with progressive reduction for at least 6 months (according to clinical evolution) +/- Azathioprine (1-3mg / kg / day) or Mycophenolate mofetila $1-2 \mathrm{~g} /$ day;

- Refractory cases: consider anti-TNF alpha or Rituximab

Figure 1. Algorithm for diagnosis and treatment of Adult primary vasculitis of central nervous system 

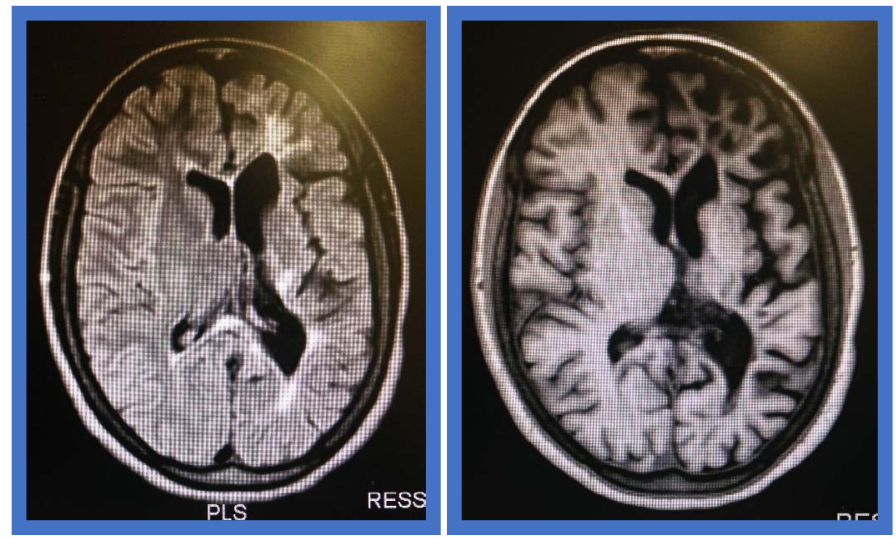

Figure 2. Primary central nervous system

construction of a diagnostic and therapeutic assistance structure. Patients undergoing unsatisfactory or inadequate care are subject to unfavorable evolution, either with death or with severe disability, which could be reversed with medication and with adequate follow-up.

The conflicting data presented by the two major studies carried out in the theme suggest that the disease may have characteristics with ethnic variations, or that it may be influenced by environmental events. Therefore, it is essential to expand the studies on the theme and its associated factors in each global region. It is possible to conclude that the best treatment should be individualized and based on the knowledge that can be extracted from the patient (clinical data, complementary tests and possible biomarkers), and the way in which it interacts with its disease, in immune-mediated diseases (Figure 2).

\section{References}

1. Cravioto H, Feigin I (1959) Noninfectious granulomatous angiitis with a predilection for the nervous system. Neurology 9: 599-609. [Crossref]

2. Salvarani C, Brown RD Jr, Christianson T, Miller DV, Giannini C, et al. (2015) An update of the Mayo Clinic cohort of patients with adult primary central nervous system vasculitis: description of 163 patients. Medicine 94: e738. [Crossref]

3. Boysson H, Zuber M, Naggara O, Neau JP, Gray F, et al. (2014) Primary angiitis of the central nervous system: description of the first fifty-two adults enrolled in the French cohort of patients with primary vasculitis of the central nervous system. Arthritis Rheumatol 66: 1315-26. [Crossref]

4. Twilt M, Benseler SM (2016) Central nervous system vasculitis in adults and children. Handb Clin Neurol 133: 283-300. [Crossref]
5. Iwase T, Ojika K, Mitake S (2001) Involvement of CD45O+ T lymphocyte infiltration in a patient with primary angiitis of the central nervous system restricted to small vessels. Eur Neurol 45: 184-185.

6. Elbers J, Halliday W, Hawkins C, Hutchinson C, Benseler SM (2010) Brain biopsy in children with primary small-vessel central nervous system vasculitis. Ann Neurol 68: 602-610. [Crossref]

7. Nagel MA, Cohrs RJ, Mahalingam R, Wellish MC, Forghani B, et al. (2008) The varicela-zoster vírus vasculopathics: clinical, CSF, imaging and virologic features. Neurology 70: 853-860. [Crossref]

8. Thomas L, Davidson M, McCluskey RT (1966) Studies of PPLO infection. The production of cerebral polyarteritis by Mycoplasma gallisepticum in turkeys; the neurotoxic property of the mycoplasma. J Exp Med 123: 897-912. [Crossref]

9. Biffi A, Greenberg SM (2011) Cerebral amyloid angiopathy: a systematic review. $J$ Clin Neurol 7: 1-9. [Crossref]

10. Salvarani C, Brown RD Jr, Hunder GG (2012) Adult primary central nervous system vasculitis. Lancet 380: 767-775. [Crossref]

11. Boysson H, Boulouis G, Aouba A (2016) Adult primary angiitis of the central nervous system: isolated small-vessel vasculitis representes distinct disease pattern. Rheumatology 262-268.

12. Salvarani C, Brown RD Jr, Calamia KT, Christianson TJ, Huston J 3rd, et al. (2001) Rapidly progressive primary central nervous system vasculitis. Rheumatology (Oxford) 50: 349-358. [Crossref]

13. Birnbaum J, Hellmann DB (2009) Primary angiitis of the central nervous system. Arch Neurol 66: 704-709. [Crossref]

14. Hajj-Ali RA, Calabrese LH (2009) Central nervous system vasculitis. Curr Opin Rheumatol 21: 10-18. [Crossref]

15. Miller DV, Salvarani C, Hunder GG, Brown RD, Parisi JE, et al. (2009) Biopsy findings in primary angiitis of the central nervous system. Am J Surg Pathol 33: 35-43. [Crossref]

16. Alrawi A, Trobe JD, Blaivas M, Musch DC (1999) Brain biopsy in primary angiitis of central nervous system. Neurology 53: 858-860. [Crossref]

17. Ferris EJ, Levine HL (1973) Cerebral arteritis: classification. Radiology 109: 327-341. [Crossref]

18. Salvarani C, Brown RD Jr, Calamia KT, Christianson TJ, Weigand SD, et al. (2007) Primary central nervous system vasculitis: analysis of 101 patients. Ann Neurol 62: 442-451. [Crossref]

19. Cupps TR, Moore PM, Fauci AS (1983) Isolated angiitis of central nervous system. Prospective diagnostic and therapeutic experience. AM J Med 74: 97-105. [Crossref]

20. Calabrese LH, Mallek JA (1988) Primary angiitis of central nervous system. Report of 8 new cases, review of literature, and proposal for diagnostic criteria. Medicine (Baltimore) 67: 20-39. [Crossref]

Copyright: (C2017 Oliveira FTM. This is an open-access article distributed under the terms of the Creative Commons Attribution License, which permits unrestricted use, distribution, and reproduction in any medium, provided the original author and source are credited. 